\section{Nitrogen and Potassium Requirements for Field-grown Areca and Mexican Fan Palms}

\author{
Timothy K. Broschat ${ }^{1}$ \\ Fort Lauderdale Research and Education Center, University of Florida, \\ 3205 College Avenue, Davie, FL 33314
}

Additional index words. Dypsis lutescens, Washingtonia robusta, potassium deficiency, fertilization, principal component analysis

\begin{abstract}
Potassium (K) deficiency is a widespread problem in palms growing in sandy or calcareous soils in southeastern United States. Its symptoms are highly conspicuous, reduce palm aesthetic appeal, are difficult to correct, and can be exacerbated by nitrogen (N) fertilization. The objective of this study was to determine the optimum fertilization rates and ratio for $\mathrm{N}$ and $\mathrm{K}$ in areca palm [Dypsis lutescens $(\mathrm{H}$. Wendl.) Beentje \& J. Dransf. and Mexican fan palm (Washingtonia robusta $\mathrm{H}$. Wendl.) growing in a calcareous sandy fill soil. Both species had their highest quality when fertilized with $12.2 \mathrm{~g} \cdot \mathrm{m}^{-2} \mathrm{~N}$ and $12.2 \mathrm{~g} \cdot \mathrm{m}^{-2} \mathrm{~K}$ from controlled-release (2-3 month release) sources every 3 months. Actual $N$ and $K$ application rates were better predictors of palm quality than $\mathrm{N}$ :K ratio at the highest fertilization rates that would be recommended for field nursery production. However, at lower application rates more typical of those used for landscape palm maintenance, palm quality improved as the N:K application ratio was decreased.
\end{abstract}

Fertilization requirements for palms growing in mineral soils differ greatly from those growing in largely organic container substrates (Broschat, 2009). Pine bark is a common component of container substrates in southeastern United States, but requires high $\mathrm{N}$ fertilization rates to compensate for $\mathrm{N}$ tie-up by microbes degrading this material (Ogden et al., 1987). Thus, fertilizers used in container production of palms and other ornamental plants contain large amounts of $\mathrm{N}$ relative to phosphorus $(\mathrm{P})$, potassium $(\mathrm{K})$, and other elements (Broschat, 2009; Poole and Conover, 1985). On the other hand, the highly leached, low cation-exchange capacity, sandy soils that predominate in the coastal plain of southeastern United States are typically deficient in $\mathrm{N}, \mathrm{K}$, magnesium $(\mathrm{Mg})$, and often micronutrients such as iron $(\mathrm{Fe})$, manganese $(\mathrm{Mn})$, and boron (B) (Tisdale and Nelson, 1975). However, there is no published research on the optimum $\mathrm{N}$ and $\mathrm{K}$ fertilization rates and ratios for ornamental palms growing in landscape soils.

Potassium deficiency, which causes foliar discoloration, leaflet necrosis, and premature senescence of the oldest leaves, is nearly ubiquitous on palms growing in these soils and has been shown to be exacerbated

\footnotetext{
This research was supported by the Florida Agricultural Experiment Station and by the USDA National Institute of Food and Agriculture Hatch project FLA-FTL-004945.

We wish to thank William Latham, Andy Warren, Andy Fu, and Susan Thor for their assistance in this project.

${ }^{1}$ Corresponding author. E-mail: tkbr@ufl.edu.
}

by high $\mathrm{N}$ fertilizers (Broschat, 1990, 2009; Mills and Jones, 1996). While N deficiency symptoms are rarely observed on palms growing in these soils, growth rate is strongly affected by $\mathrm{N}$ fertilization rates. Thus, in field nurseries, where both visual quality and rapid growth are important, $\mathrm{N}$ and $\mathrm{K}$ fertilization rates must be properly balanced. It is not known if absolute amounts of $\mathrm{N}$ and $\mathrm{K}$ are critical for optimum growth and quality of field-grown palms or whether the ratio of these two elements is more important.

Since K deficiency was first identified as a serious problem in landscape and fieldgrown palms in southeastern United States in the late 1980s (Broschat, 1990), sequential trials with fertilizers containing increasingly lower $\mathrm{N}: \mathrm{K}$ ratios and higher proportions of controlled-release $\mathrm{K}$ culminated in the recommendation of products having an analysis of $8 \mathrm{~N}-0.9 \mathrm{P}-10 \mathrm{~K}-4 \mathrm{Mg}$ plus $2 \%$ of $\mathrm{Mn}$ and $\mathrm{Fe}$ and trace amounts of zinc $(\mathrm{Zn})$, copper $(\mathrm{Cu})$, and B (Broschat, 2009).

Because of the severity of K deficiency on these soils, the need to reduce leaching losses, and the need to balance the ratios of $\mathrm{N}, \mathrm{K}$, and $\mathrm{Mg}$ over time, Broschat (2009) recommended that $100 \%$ of the $\mathrm{N}, \mathrm{K}$, and $\mathrm{Mg}$ should be in controlled-release form. Although a landscape maintenance application rate for this $8 \mathrm{~N}-0.9 \mathrm{P}-10 \mathrm{~K}-4 \mathrm{Mg}$ formulation of $73 \mathrm{~g} \cdot \mathrm{m}^{-2}$ of canopy or landscape area every 3 months has been recommended (Broschat, 2014), there is no research showing what the optimum fertilization rates are for either landscape maintenance or field production of palms. Commercial field production application rates of this product are typically twice the recommended landscape maintenance rates (Broschat, 2009). The purpose of this study was to determine the optimum $\mathrm{N}$ and $\mathrm{K}$ fertilization rates and ratios for field-grown areca and Mexican fan palms in southeastern Florida.

\section{Materials and Methods}

Areca and Mexican fan palms grown in 2.8-L containers were planted on 1 Nov. 2008 and 12 Jan. 2009, respectively into a field of Margate fine sand soil (siliceous, hyperthermic, and Mollic psammaquent) overlaid $\approx 40$ years earlier with a calcareous sandy subsoil fill soil dredged from nearby ponds. Nine representative soils samples had an average $\mathrm{pH}$ of $7.0,3.4 \%$ organic matter, and were noted as having very high $\mathrm{Ca}$ (1355 ppm), but low to very low levels of Bray1-P (3.6 ppm), K (8.8 ppm), Mg (26.1 ppm), Na (19.2 ppm), S (15.1 ppm), Zn (0.1 ppm), Mn (1.0 ppm), Cu (0.1 ppm), and B (1.0 ppm) (A \& L Southern Laboratories, Deerfield Beach, FL). This site was selected as having soils similar to the calcareous sandy fill soils on which most urban and suburban landscapes in south Florida are planted.

Eighty-one plots (blocks) each containing three palms of both species spaced $3 \mathrm{~m}$ apart in rows, were separated from each other by an additional $3 \mathrm{~m}$ space to prevent roots from entering adjacent plots. Twenty-seven treatments were applied to three replicate plots in a randomized complete block design with

Table 1. Nitrogen and potassium rates (per application) used for areca (Dypsis lutescens) and Mexican fan palms (Washingtonia robusta) growing in a calcareous sandy fill soil in Fort Lauderdale, FL.

\begin{tabular}{|c|c|c|c|}
\hline Treatment & $\mathrm{N}\left(\mathrm{g} \cdot \mathrm{m}^{-2}\right)$ & $\mathrm{K}\left(\mathrm{g} \cdot \mathrm{m}^{-2}\right)$ & $\mathrm{N}: \mathrm{K}$ ratio \\
\hline 1 & 2.44 & 2.44 & 1.0 \\
\hline 2 & 2.44 & 4.88 & 0.5 \\
\hline 3 & 2.44 & 7.32 & 0.33 \\
\hline 4 & 2.44 & 9.76 & 0.25 \\
\hline 5 & 2.44 & 12.20 & 0.2 \\
\hline 6 & 4.88 & 4.88 & 1.0 \\
\hline 7 & 4.88 & 7.32 & 0.67 \\
\hline 8 & 4.88 & 9.76 & 0.5 \\
\hline 9 & 4.88 & 12.20 & 0.4 \\
\hline 10 & 4.88 & 14.64 & 0.33 \\
\hline 11 & 7.34 & 7.34 & 1.0 \\
\hline 12 & 7.34 & 9.76 & 0.6 \\
\hline 13 & 7.34 & 12.20 & 0.6 \\
\hline 14 & 7.34 & 14.64 & 0.5 \\
\hline 15 & 7.34 & 19.82 & 0.37 \\
\hline 16 & 9.78 & 7.32 & 1.33 \\
\hline 17 & 9.78 & 9.78 & 1.0 \\
\hline 18 & 9.78 & 12.20 & 0.8 \\
\hline 19 & 9.78 & 14.64 & 0.67 \\
\hline 20 & 9.78 & 19.56 & 0.5 \\
\hline 21 & 12.21 & 9.78 & 1.33 \\
\hline 22 & 12.21 & 12.20 & 1.0 \\
\hline 23 & 12.21 & 14.64 & 0.5 \\
\hline 24 & 12.21 & 19.56 & 0.62 \\
\hline 25 & 12.21 & 24.42 & 0.5 \\
\hline $26^{z}$ & 0.00 & 0.00 & \\
\hline $27^{y}$ & 0.00 & 0.00 & \\
\hline
\end{tabular}

${ }^{\mathrm{z}}$ Absolute control (no fertilizer applied).

${ }^{\mathrm{y}}$ Only phosphorus and micronutrients applied. 
individual palms $(n=9)$ as the experimental units. Palms were irrigated every other day for the first 6 months and every $3 \mathrm{~d}$ thereafter with $2 \mathrm{~cm}$ of water per application using overhead rotary irrigation heads mounted on 2-m tall risers. All palms received $18 \mathrm{~g}$ of Osmocote $19 \mathrm{~N}-2.6 \mathrm{P}-10 \mathrm{~K}$ plus micronutrients (8-9 month release at $20{ }^{\circ} \mathrm{C}$; Everris, Marysville, $\mathrm{OH}$ ) at planting and 3 months later to aid in establishment. Experimental fertilizer treatments were applied on 22 Oct. 2009 and every 3 months thereafter as shown in Table 1. All palms except those in treatment 26 (control receiving no fertilizer) also received $7.3 \mathrm{~g}$ of $\mathrm{P}$ from triple superphosphate (Helena Chemical Co., Fort Pierce, FL), $0.11 \mathrm{~g}$ of Fe

Table 2. Correlations of original plant quality variables with the first principal component (PC1) for areca palm (Dypsis lutescens) and Mexican fan palm (Washingtonia robusta) growing in a calcareous sandy fill soil in Fort Lauderdale, FL.

\begin{tabular}{lcc}
\hline Variable & $\begin{array}{c}\text { PC1 (areca } \\
\text { palm) }\end{array}$ & $\begin{array}{c}\text { PC1 (Mexican } \\
\text { fan palm) }\end{array}$ \\
\hline Height & 0.39 & 0.41 \\
No. of leaves & 0.43 & 0.41 \\
No. of stems & 0.30 & \\
N rating & 0.51 & 0.47 \\
K rating & 0.44 & 0.46 \\
Mg rating & 0.33 & 0.47 \\
Eigenvalue & 3.36 & 2.73 \\
Proportion of & 0.56 & 0.55 \\
$\quad$ variance & & \\
\hline
\end{tabular}

Table 3. Height and overall quality scores [first principal component (PC1) scores] for areca palm (Dypsis lutescens) and Mexican fan palm (Washingtonia robusta) fertilized with various combinations of $\mathrm{N}$ and $\mathrm{K}$ fertilization rates. Palms were grown on a calcareous sandy fill soil in Fort Lauderdale, FL $(n=9)$.

\begin{tabular}{|c|c|c|c|c|c|c|}
\hline \multirow[b]{2}{*}{ Treatment } & \multirow[b]{2}{*}{$\begin{array}{l}\text { PC1 } \\
\text { score }\end{array}$} & \multicolumn{2}{|c|}{ Areca palm } & \multicolumn{3}{|c|}{ Mexican fan palm } \\
\hline & & $\begin{array}{l}\mathrm{Ht} \\
(\mathrm{cm})\end{array}$ & $\begin{array}{l}\text { PC1 score } \\
\text { rank }\end{array}$ & $\begin{array}{l}\text { PC1 } \\
\text { score }\end{array}$ & $\begin{array}{c}\mathrm{Ht} \\
(\mathrm{cm})\end{array}$ & $\begin{array}{l}\text { PC1 score } \\
\text { rank }\end{array}$ \\
\hline 1 & $82.4 c-i^{z}$ & $183.4 \mathrm{c}-\mathrm{j}$ & 17 & $37.8 \mathrm{ijk}$ & $71.3 \mathrm{ijk}$ & 23 \\
\hline 2 & $78.1 \mathrm{~d}-\mathrm{i}$ & $177.6 \mathrm{e}-\mathrm{j}$ & 19 & $43.9 \mathrm{f}-\mathrm{k}$ & $83.2 \mathrm{e}-\mathrm{k}$ & 19 \\
\hline 3 & $70.3 \mathrm{hi}$ & $158.7 \mathrm{ij}$ & 24 & $33.4 \mathrm{k}$ & $60.0 \mathrm{kl}$ & 26 \\
\hline 4 & $77.4 \mathrm{e}-\mathrm{i}$ & $175.7 \mathrm{f}-\mathrm{j}$ & 20 & $39.3 \mathrm{~h}-\mathrm{k}$ & 72.7 h-k & 22 \\
\hline 5 & $66.2 \mathrm{i}$ & $148.1 \mathrm{j}$ & 25 & $46.7 \mathrm{e}-\mathrm{k}$ & $89.1 \mathrm{~d}-\mathrm{k}$ & 18 \\
\hline 6 & $75.7 \mathrm{f}-\mathrm{i}$ & $172.3 \mathrm{~g}-\mathrm{j}$ & 22 & $53.6 \mathrm{c}-\mathrm{g}$ & $105.1 \mathrm{c}-\mathrm{f}$ & 11 \\
\hline 7 & $84.6 \mathrm{~b}-\mathrm{h}$ & $193.9 \mathrm{~b}-\mathrm{i}$ & 16 & $40.2 \mathrm{~g}-\mathrm{k}$ & $74.1 \mathrm{~g}-\mathrm{k}$ & 21 \\
\hline 8 & $90.0 \mathrm{~b}-\mathrm{g}$ & $207.1 \mathrm{~b}-\mathrm{h}$ & 12 & $49.7 \mathrm{~d}-\mathrm{j}$ & $96.9 \mathrm{c}-\mathrm{j}$ & 16 \\
\hline 9 & $88.5 \mathrm{~b}-\mathrm{f}$ & $204.1 \mathrm{~b}-\mathrm{h}$ & 14 & $51.3 \mathrm{c}-\mathrm{i}$ & $99.1 \mathrm{c}-\mathrm{i}$ & 15 \\
\hline 10 & $87.9 \mathrm{~b}-\mathrm{h}$ & 200.4 b-i & 15 & $52.9 \mathrm{c}-\mathrm{h}$ & $103.3 \mathrm{c}-\mathrm{g}$ & 12 \\
\hline 11 & $95.3 \mathrm{bcd}$ & 219.8 b-e & 6 & $57.5 \mathrm{c}-\mathrm{f}$ & $113.1 \mathrm{~cd}$ & 8 \\
\hline 12 & $93.9 \mathrm{~b}-\mathrm{e}$ & $217.0 \mathrm{~b}-\mathrm{f}$ & 8 & 60.2 cde & $118.2 \mathrm{~cd}$ & 6 \\
\hline 13 & 92.3 b-f & $211.6 \mathrm{~b}-\mathrm{g}$ & 9 & $59.5 \mathrm{cde}$ & $116.6 \mathrm{~cd}$ & 7 \\
\hline 14 & $73.2 \mathrm{~g}-\mathrm{j}$ & $166.1 \mathrm{hij}$ & 23 & $36.7 \mathrm{jk}$ & $67.5 \mathrm{jk}$ & 24 \\
\hline 15 & $81.7 \mathrm{c}-\mathrm{i}$ & $185.6 \mathrm{~d}-\mathrm{j}$ & 18 & $52.2 \mathrm{c}-\mathrm{h}$ & $101.4 \mathrm{c}-\mathrm{h}$ & 13 \\
\hline 16 & $76.4 \mathrm{f}-\mathrm{i}$ & $173.5 \mathrm{ghi}$ & 21 & $40.7 \mathrm{~g}-\mathrm{k}$ & $76.0 \mathrm{f}-\mathrm{k}$ & 20 \\
\hline 17 & $90.1 \mathrm{~b}-\mathrm{g}$ & $206.7 \mathrm{~b}-\mathrm{h}$ & 11 & $46.8 \mathrm{e}-\mathrm{k}$ & 89.2 d-k & 17 \\
\hline 18 & $94.8 \mathrm{bcd}$ & $219.1 \mathrm{~b}-\mathrm{e}$ & 7 & $56.6 \mathrm{cde}$ & $111.6 \mathrm{cde}$ & 9 \\
\hline 19 & $98.7 \mathrm{bc}$ & $227.4 \mathrm{bcd}$ & 4 & $78.6 \mathrm{a}$ & $158.0 \mathrm{a}$ & 2 \\
\hline 20 & $101.8 \mathrm{ab}$ & $235.9 \mathrm{ab}$ & 2 & $63.4 \mathrm{bc}$ & $125.4 \mathrm{bc}$ & 4 \\
\hline 21 & $98.7 \mathrm{bc}$ & $228.7 \mathrm{bc}$ & 5 & $51.3 \mathrm{c}-\mathrm{i}$ & $98.5 \mathrm{c}-\mathrm{i}$ & 14 \\
\hline 22 & $117.3 \mathrm{a}$ & $274.7 \mathrm{a}$ & 1 & $81.9 \mathrm{a}$ & $166.6 \mathrm{a}$ & 1 \\
\hline 23 & $88.6 \mathrm{~b}-\mathrm{g}$ & 202.9 b-h & 13 & $62.7 \mathrm{~cd}$ & $123.9 \mathrm{c}$ & 5 \\
\hline 24 & $90.7 \mathrm{~b}-\mathrm{f}$ & $208.0 \mathrm{~b}-\mathrm{h}$ & 10 & $56.1 \mathrm{c}-\mathrm{f}$ & $109.8 \mathrm{cde}$ & 10 \\
\hline 25 & $100.8 \mathrm{ab}$ & $233.6 \mathrm{ab}$ & 3 & $76.4 \mathrm{ab}$ & $154.8 \mathrm{ab}$ & 3 \\
\hline 26 & $45.4 \mathrm{j}$ & $101.6 \mathrm{k}$ & 27 & 18.71 & 30.91 & 27 \\
\hline 27 & $47.2 \mathrm{j}$ & $102.9 \mathrm{k}$ & 26 & $35.4 \mathrm{k}$ & $65.0 \mathrm{k}$ & 25 \\
\hline$P$ value & $<0.0001$ & $<0.0001$ & & $<0.0001$ & $<0.0001$ & \\
\hline
\end{tabular}

${ }^{\mathrm{z}}$ Mean separation within columns by the Waller-Duncan k-ratio method, $P=0.05$.

from Trachelene Fe (=FeEDTA + FeDTPA) (QC Corp., Baltimore, MD), $1.47 \mathrm{~g}$ of $\mathrm{Mn}$ from manganese sulfate (Industrias Sulfamex, Pánuco, Mexico), $0.11 \mathrm{~g}$ of $\mathrm{B}$ from Granubor (US Borax, Valencia, CA), $0.04 \mathrm{~g}$ of $\mathrm{Cu}$ from $\mathrm{CuSO}_{4}$ (Fisher Scientific, Fair Lawn, NJ), and $0.04 \mathrm{~g}$ of $\mathrm{Zn}$ from $\mathrm{ZnSO}_{4}$ (Fisher Scientific) at each fertilization to prevent these elements from limiting growth or quality. All fertilizers were applied by broadcasting the materials uniformly over a $1 \mathrm{~m}^{2}$ area surrounding each palm. Magnesium was applied as kieserite (Kali and Salz, Kassel, Germany) at a rate equal to $40 \%$ of the $\mathrm{K}$ application rate to prevent $\mathrm{K}: \mathrm{Mg}$ imbalances (Broschat, 2009; von Uexkull and Fairhurst, 1991).

The Bahiagrass (Paspalum notatum Flügge.) growing between the plots was mowed at a height of $10 \mathrm{~cm}$ as needed while weeds growing within $60 \mathrm{~cm}$ of each palm were controlled by spraying with glyphosate. Plants were measured for total height, the number of leaves and stems (areca palms only) were counted, and each plant was rated for severity of $\mathrm{N}, \mathrm{K}$, and $\mathrm{Mg}$ deficiencies (30 Nov. 2012). Deficiency severity ratings were based on a $0-5$ scale with $0=$ dead from a nutrient deficiency, $1=$ severely deficient, $3=$ moderately deficient, and $5=$ no deficiency symptoms present. Leaf samples consisting of the central leaflets of the youngest fully expanded leaf on each palm were also collected at that time. Samples from the three palms of each species in each plot were pooled for analysis scores were not statistically significant. ANOVA and mean separation of the treatment PC1 scores showed that treatment 22 resulted in the highest quality palms for both species, although treatments 20 and 25 had statistically equivalent quality for areca palm and treatments 19 and 25 were equally good for Mexican fan palm (Table 3). All of these treatments had high rates of $\mathrm{N}$ with $\mathrm{N}: \mathrm{K}$ ratios generally between 0.5 and 1 . Equivalent high quality could be achieved by using slightly lower $\mathrm{N}$ rates, but higher $\mathrm{K}$ rates in both species (Figs. 1 and 2). The fact that treatments 23 and 24 performed significantly poorer than treatments having similar $\mathrm{N}$ rates and higher or lower $\mathrm{K}$ rates may be due to chance assignment of one or more of those treatment plots to areas in the field having poorer soil.

Optimum growth and quality was dependent on high levels of both $\mathrm{N}$ and $\mathrm{K}$, and high $\mathrm{N}$ rates without equivalent $\mathrm{K}$ rates resulted in a sharp reduction in quality (Fig. 1). Similarly, high $\mathrm{K}$ rates with low $\mathrm{N}$ rates produced poor quality palms. In African oil palm (Elaeis guineensis Jacq.), increases in $\mathrm{K}$ fertilization did not increase yield or vegetative dry matter unless $\mathrm{N}$ fertilization rates were also increased (von Uexkull and Fairhurst, 1991). 


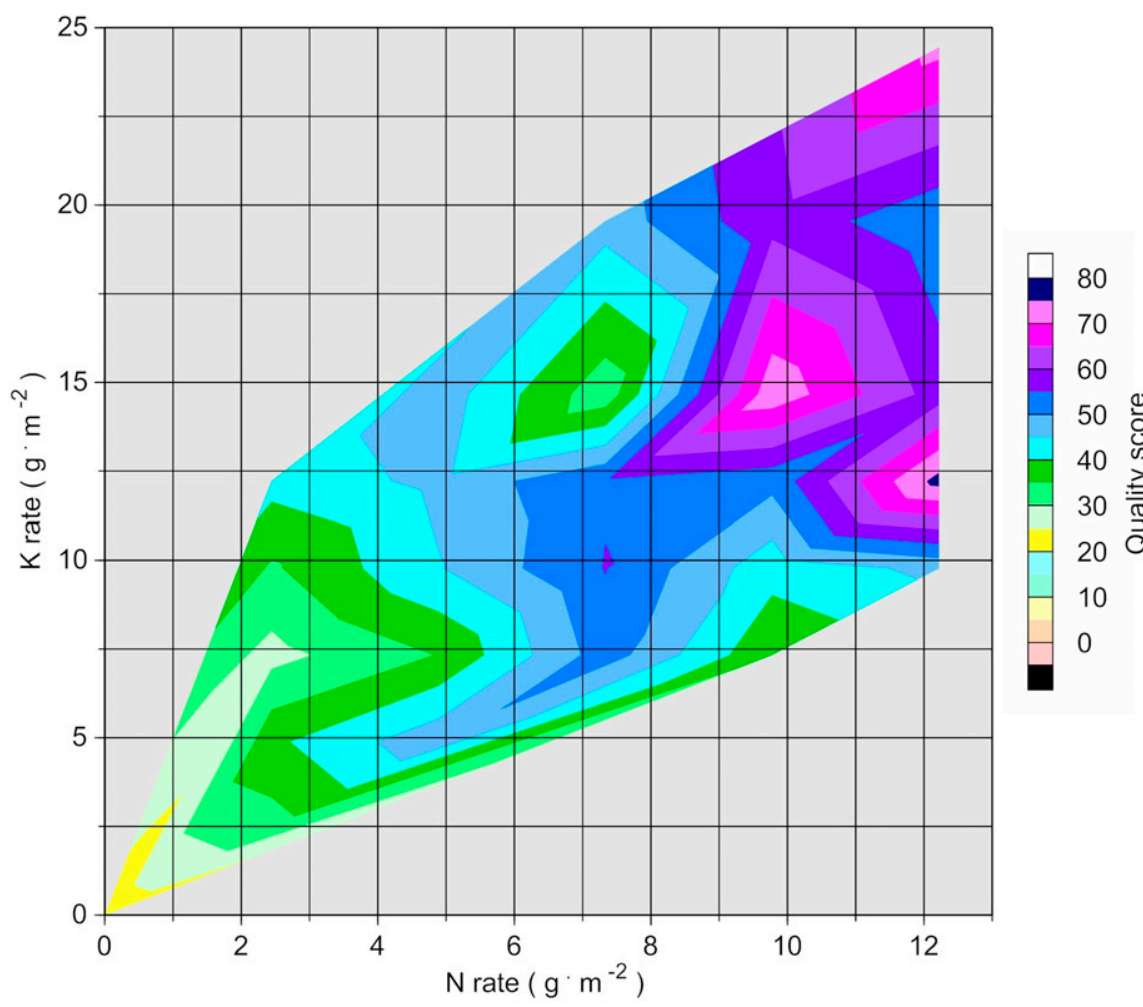

Fig. 1. Effects of $\mathrm{N}$ and $\mathrm{K}$ fertilization rates on overall quality (first principal component) scores of areca palms (Dypsis lutescens) growing in a calcareous sandy fill soil in Fort Lauderdale, FL.

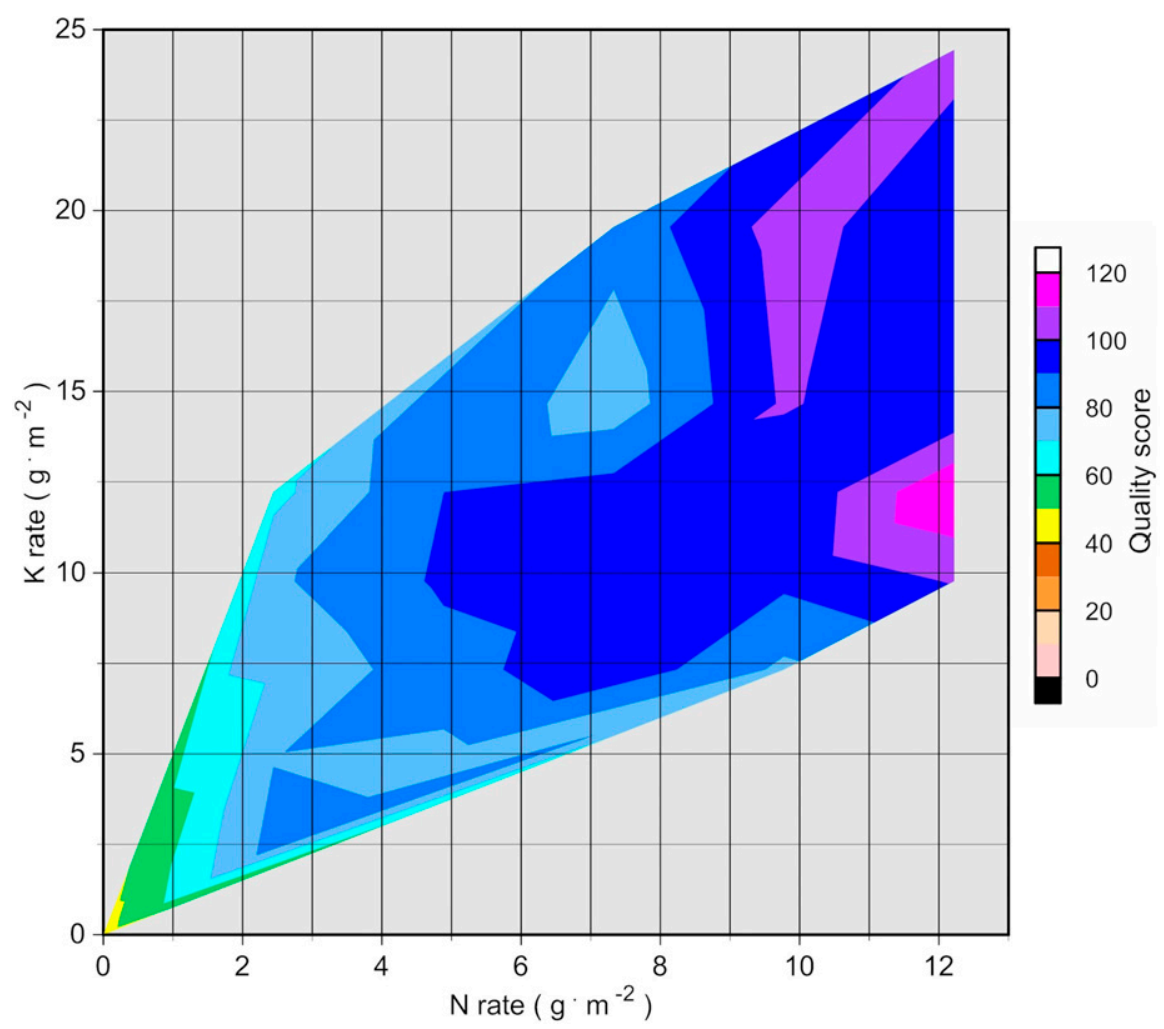

Fig. 2. Effects of $\mathrm{N}$ and $\mathrm{K}$ fertilization rates on overall quality (first principal component) scores of Mexican fan palms (Washingtonia robusta) growing in a calcareous sandy fill soil in Fort Lauderdale, FL.

In comparing the response plots (Figs. 1 and 2) for areca and Mexican fan palms, it is apparent that Mexican fan palms were much less responsive to $\mathrm{N}$ and $\mathrm{K}$ fertilization rates than areca palms. That is, Mexican fan palms generally exhibited higher PC1 scores than areca palms at equivalent fertilization rates. While both species exhibited $\mathrm{K}$ deficiency symptoms, areca palm visual quality was also affected by $\mathrm{N}$ fertilization rate.

The difference in quality in Mexican fan palms, but not in areca palms between treatment 26, which received no fertilizer, and treatment 27 , which received only $\mathrm{P}$ plus micronutrients suggests that growth and quality in these Mexican fan palms was limited by $\mathrm{P}$ deficiency as no micronutrient deficiency symptoms were observed in this species. Unfertilized palms (treatments 26 and 27) of both species were extremely stunted or dead after 3 years, whereas few of the fertilized palms died during that time. Phosphorus deficiency is rare in palms in the United States, but can be locally severe, especially on reclaimed Everglades soils such as those found in this study area (Broschat and Elliott, 2009). While P fertilization was essential for Mexican fan palms, areca palms did not respond to supplemental $\mathrm{P}$ in this study or in another on a nearby plot (Broschat, 2015).

Although rapid growth is a primary concern for nursery production of palms, plant height was highly correlated with overall plant quality (PC1 scores) (Table 2) and rankings of the treatment means for $\mathrm{PC} 1$ scores and plant height were virtually identical for both species (Table 3). Thus, the largest plants also tended to have the highest nutrient deficiency ratings (fewest visual symptoms) and the greatest number of leaves.

Nitrogen and $\mathrm{K}$ fertilization treatments did not affect leaf concentrations of $\mathrm{N}, \mathrm{P}$, or $\mathrm{Mg}$ in areca palms, but significantly affected $\mathrm{K}$ concentrations (Table 4). Treatment 22, which resulted in the highest quality scores, had some of the lowest foliar $\mathrm{K}$ concentrations. This was likely due to a dilution effect. On the other hand, treatments 20 and 25 , which had statistically equivalent quality scores to treatment 22 , had some of the highest $\mathrm{K}$ concentrations. Treatments 20 and 25 had higher $\mathrm{K}$ application rates $\left(19.56\right.$ and $24.42 \mathrm{~g} \cdot \mathrm{m}^{-2}$, respectively) than treatment $22\left(12.2 \mathrm{~g} \cdot \mathrm{m}^{-2}\right)$, but similar, or only slightly lower $\mathrm{N}$ application rates.

Phosphorus, $\mathrm{K}$, and $\mathrm{Mg}$ concentrations in areca palms were positively influenced by $\mathrm{K}$ fertilization rate, while $\mathrm{N}$ fertilization rate had negative, though generally nonsignificant effects on leaf $\mathrm{N}, \mathrm{P}, \mathrm{K}$, and $\mathrm{Mg}$ concentrations (Table 6). Since $\mathrm{K}$ and $\mathrm{Mg}$ application rates were maintained at a constant 2.5:1 ratio to prevent $\mathrm{K}: \mathrm{Mg}$ imbalances, higher $\mathrm{K}$ application rates also resulted in higher $\mathrm{Mg}$ rates.

For Mexican fan palms, $\mathrm{N}$ and $\mathrm{K}$ application rates had no effect on leaf $\mathrm{N}$ concentrations, but significantly affected $\mathrm{P}, \mathrm{K}$, and $\mathrm{Mg}$ concentrations in the foliage (Table 5). Since N fertilization tends to stimulate growth, any increase in $\mathrm{N}$ uptake will be offset by dilution within the larger canopy resulting from $\mathrm{N}$ fertilization (von Uexkull and Fairhurst, 1991). Foliar P concentrations were highest in treatment 1 , which had the 
Table 4. Leaf nutrient concentrations for areca palms (Dypsis lutescens) fertilized with various combinations of $\mathrm{N}$ and $\mathrm{K}$ fertilization rates. Palms were grown in a calcareous fill soil in Fort Lauderdale, FL $(n=3)$.

\begin{tabular}{|c|c|c|c|c|c|c|}
\hline \multirow[b]{2}{*}{ Treatment } & \multirow{2}{*}{$\begin{array}{l}\mathrm{N} \text { rate } \\
\left(\mathrm{g} \cdot \mathrm{m}^{-2}\right)\end{array}$} & \multirow{2}{*}{$\begin{array}{l}\mathrm{K} \text { rate } \\
\left(\mathrm{g} \cdot \mathrm{m}^{-2}\right)\end{array}$} & \multicolumn{4}{|c|}{ Leaf nutrient concn } \\
\hline & & & $\mathrm{N}(\%)$ & $\mathrm{P}(\%)$ & K (\%) & $\operatorname{Mg}(\%)$ \\
\hline 1 & 2.44 & 2.44 & 1.02 & 0.12 & $0.73 \mathrm{a}-\mathrm{f}$ & 0.155 \\
\hline 2 & 2.44 & 4.88 & 1.3 & 0.09 & $0.62 \mathrm{~d}-\mathrm{g}$ & 0.161 \\
\hline 3 & 2.44 & 7.32 & 1.21 & 0.12 & $0.77 \mathrm{a}-\mathrm{e}$ & 0.177 \\
\hline 4 & 2.44 & 9.76 & 1.19 & 0.11 & $0.70 \mathrm{~b}-\mathrm{f}$ & 0.157 \\
\hline 5 & 2.44 & 12.20 & 1.05 & 0.13 & $0.95 \mathrm{a}$ & 0.181 \\
\hline 6 & 4.88 & 4.88 & 1.25 & 0.12 & $0.63 \mathrm{c}-\mathrm{g}$ & 0.167 \\
\hline 7 & 4.88 & 7.32 & 1.31 & 0.12 & $0.75 \mathrm{a}-\mathrm{f}$ & 0.173 \\
\hline 8 & 4.88 & 9.76 & 1.44 & 0.14 & $0.66 \mathrm{~b}-\mathrm{g}$ & 0.157 \\
\hline 9 & 4.88 & 12.20 & 1.2 & 0.11 & $0.69 \mathrm{~b}-\mathrm{g}$ & 0.152 \\
\hline 10 & 4.88 & 14.64 & 1.18 & 0.11 & $0.88 \mathrm{abc}$ & 0.188 \\
\hline 11 & 7.34 & 7.34 & 1.26 & 0.1 & $0.56 \mathrm{efg}$ & 0.153 \\
\hline 12 & 7.34 & 9.76 & 1.3 & 0.11 & $0.62 \mathrm{~d}-\mathrm{g}$ & 0.147 \\
\hline 13 & 7.34 & 12.20 & 1.2 & 0.1 & $0.68 \mathrm{~b}-\mathrm{g}$ & 0.16 \\
\hline 14 & 7.34 & 14.64 & 1.15 & 0.11 & $0.82 \mathrm{a}-\mathrm{d}$ & 0.194 \\
\hline 15 & 7.34 & 19.82 & 1.26 & 0.12 & $0.85 \mathrm{a}-\mathrm{d}$ & 0.195 \\
\hline 16 & 9.78 & 7.32 & 1.06 & 0.1 & $0.65 \mathrm{c}-\mathrm{g}$ & 0.176 \\
\hline 17 & 9.78 & 9.78 & 1.16 & 0.1 & $0.64 \mathrm{c}-\mathrm{g}$ & 0.162 \\
\hline 18 & 9.78 & 12.20 & 1.06 & 0.11 & $0.79 \mathrm{a}-\mathrm{e}$ & 0.168 \\
\hline 19 & 9.78 & 14.64 & 1.22 & 0.1 & 0.80 a-e & 0.161 \\
\hline 20 & 9.78 & 19.56 & 1.32 & 0.1 & $0.97 \mathrm{a}$ & 0.168 \\
\hline 21 & 12.21 & 9.78 & 1.28 & 0.09 & $0.46 \mathrm{gh}$ & 0.15 \\
\hline 22 & 12.21 & 12.20 & 1.42 & 0.08 & $0.52 \mathrm{fgh}$ & 0.14 \\
\hline 23 & 12.21 & 14.64 & 1.25 & 0.09 & $0.79 \mathrm{a}-\mathrm{d}$ & 0.163 \\
\hline 24 & 12.21 & 19.56 & 1.25 & 0.11 & $0.87 \mathrm{abc}$ & 0.187 \\
\hline 25 & 12.21 & 24.42 & 1.28 & 0.11 & $0.90 \mathrm{ab}$ & 0.192 \\
\hline 26 & 0.00 & 0.00 & 1.41 & 0.08 & $0.66 \mathrm{~b}-\mathrm{g}$ & 0.155 \\
\hline 27 & 0.00 & 0.00 & 1.2 & 0.1 & $0.30 \mathrm{~h}$ & 0.134 \\
\hline$P$ value & & & NS & NS & $<0.0001$ & NS \\
\hline
\end{tabular}

NS $=$ nonsignificant

Table 5. Leaf nutrient concentrations for Mexican fan palms (Washingtonia robusta) fertilized with various combinations of $\mathrm{N}$ and $\mathrm{K}$ fertilizer rates. Palms were grown in a calcareous sandy fill soil in Fort Lauderdale, FL $(n=3)$.

\begin{tabular}{|c|c|c|c|c|c|c|}
\hline \multirow[b]{2}{*}{ Treatment } & \multirow{2}{*}{$\begin{array}{l}\mathrm{N} \text { rate } \\
\left(\mathrm{g} \cdot \mathrm{m}^{-2}\right)\end{array}$} & \multirow{2}{*}{$\begin{array}{l}\mathrm{K} \text { rate } \\
\left(\mathrm{g} \cdot \mathrm{m}^{-2}\right)\end{array}$} & \multicolumn{4}{|c|}{ Leaf nutrient concn } \\
\hline & & & $\mathrm{N}(\%)$ & P (\%) & K (\%) & $\operatorname{Mg}(\%)$ \\
\hline 1 & 2.44 & 2.44 & 2.00 & $0.30 \mathrm{a}$ & $0.64 \mathrm{~g}$ & $0.138 \mathrm{~cd}$ \\
\hline 2 & 2.44 & 4.88 & 1.52 & $0.20 \mathrm{bc}$ & $1.29 \mathrm{def}$ & $0.142 \mathrm{~cd}$ \\
\hline 3 & 2.44 & 7.32 & 1.55 & $0.21 \mathrm{bc}$ & $1.54 \mathrm{a}-\mathrm{e}$ & $0.167 \mathrm{bc}$ \\
\hline 4 & 2.44 & 9.76 & 1.62 & $0.21 \mathrm{bc}$ & $1.87 \mathrm{a}$ & $0.132 \mathrm{~cd}$ \\
\hline 5 & 2.44 & 12.20 & 1.61 & $0.22 \mathrm{bc}$ & $1.57 \mathrm{a}-\mathrm{e}$ & $0.145 \mathrm{bcd}$ \\
\hline 6 & 4.88 & 4.88 & 1.64 & $0.22 \mathrm{bc}$ & $1.24 \mathrm{ef}$ & $0.136 \mathrm{~cd}$ \\
\hline 7 & 4.88 & 7.32 & 1.56 & $0.23 \mathrm{~b}$ & $1.57 \mathrm{a}-\mathrm{e}$ & $0.131 \mathrm{~d}$ \\
\hline 8 & 4.88 & 9.76 & 1.77 & $0.21 \mathrm{bc}$ & $1.40 \mathrm{~b}-\mathrm{f}$ & $0.125 \mathrm{~d}$ \\
\hline 9 & 4.88 & 12.20 & 1.54 & $0.21 \mathrm{bc}$ & $1.44 \mathrm{~b}-\mathrm{f}$ & $0.136 \mathrm{~cd}$ \\
\hline 10 & 4.88 & 14.64 & 1.67 & $0.22 \mathrm{bc}$ & $1.54 \mathrm{a}-\mathrm{e}$ & $0.152 \mathrm{bcd}$ \\
\hline 11 & 7.34 & 7.34 & 1.96 & $0.24 \mathrm{~b}$ & $1.24 \mathrm{ef}$ & $0.138 \mathrm{~cd}$ \\
\hline 12 & 7.34 & 9.76 & 1.71 & $0.21 \mathrm{bc}$ & $1.14 \mathrm{f}$ & $0.142 \mathrm{~cd}$ \\
\hline 13 & 7.34 & 12.20 & 1.72 & $0.21 \mathrm{bc}$ & $1.29 \mathrm{def}$ & $0.142 \mathrm{~cd}$ \\
\hline 14 & 7.34 & 14.64 & 1.54 & $0.19 \mathrm{bc}$ & $1.47 \mathrm{~b}-\mathrm{f}$ & $0.149 \mathrm{bcd}$ \\
\hline 15 & 7.34 & 19.82 & 1.69 & $0.21 \mathrm{bc}$ & $1.64 \mathrm{a}-\mathrm{d}$ & $0.142 \mathrm{~cd}$ \\
\hline 16 & 9.78 & 7.32 & 1.72 & $0.21 \mathrm{bc}$ & $1.20 \mathrm{ef}$ & $0.142 \mathrm{~cd}$ \\
\hline 17 & 9.78 & 9.78 & 1.65 & $0.21 \mathrm{bc}$ & $1.39 \mathrm{c}-\mathrm{f}$ & $0.126 \mathrm{~d}$ \\
\hline 18 & 9.78 & 12.20 & 1.66 & $0.22 \mathrm{bc}$ & $1.42 \mathrm{~b}-\mathrm{f}$ & $0.141 \mathrm{~cd}$ \\
\hline 19 & 9.78 & 14.64 & 1.72 & $0.21 \mathrm{bc}$ & $1.44 \mathrm{~b}-\mathrm{f}$ & $0.144 \mathrm{bcd}$ \\
\hline 20 & 9.78 & 19.56 & 1.56 & $0.20 \mathrm{bc}$ & $1.67 \mathrm{abc}$ & $0.142 \mathrm{~cd}$ \\
\hline 21 & 12.21 & 9.78 & 1.63 & $0.18 \mathrm{bc}$ & $1.40 \mathrm{~b}-\mathrm{f}$ & $0.133 \mathrm{~cd}$ \\
\hline 22 & 12.21 & 12.20 & 1.69 & $0.19 \mathrm{bc}$ & $1.23 \mathrm{ef}$ & $0.152 \mathrm{bcd}$ \\
\hline 23 & 12.21 & 14.64 & 1.6 & $0.20 \mathrm{bc}$ & $1.56 \mathrm{a}-\mathrm{e}$ & $0.141 \mathrm{~cd}$ \\
\hline 24 & 12.21 & 19.56 & 1.55 & $0.21 \mathrm{bc}$ & $1.76 \mathrm{ab}$ & $0.147 \mathrm{bcd}$ \\
\hline 25 & 12.21 & 24.42 & 1.6 & $0.20 \mathrm{bc}$ & $1.48 \mathrm{~b}-\mathrm{f}$ & $0.136 \mathrm{~cd}$ \\
\hline 26 & 0.00 & 0.00 & 1.86 & $0.17 \mathrm{c}$ & $1.21 \mathrm{ef}$ & $0.240 \mathrm{a}$ \\
\hline 27 & 0.00 & 0.00 & 1.53 & $0.19 \mathrm{bc}$ & $0.65 \mathrm{~g}$ & $0.178 \mathrm{~b}$ \\
\hline$P$ value & & & NS & 0.044 & $<0.0001$ & 0.0003 \\
\hline
\end{tabular}

NS $=$ nonsignificant.

lowest application rates for both $\mathrm{N}$ and $\mathrm{K}$. These palms were among the smallest and had the lowest quality scores of any fertilized treatment. Potassium concentrations in the leaves of Mexican fan palms were strongly affected by treatment. However, as with areca palms, the treatments that produced the highest quality and largest size (treatments 19, 22, and 25) did not have the highest foliar K concentrations. Potassium fertilization in coconut (Cocos nucifera) increased yield, but not foliar K concentrations (Manciot et al., 1979). Treatment 4, which had the highest foliar K concentration, had the lowest $\mathrm{N}$ application rate, and one of the lowest $\mathrm{N}$ : $\mathrm{K}$ ratios (Table 1). Other treatments with high foliar $\mathrm{K}$ concentrations such as treatments 20 and 24 had high $\mathrm{N}$ and $\mathrm{K}$ application rates, but also relatively low $\mathrm{N}: \mathrm{K}$ ratios. In general, leaf $\mathrm{K}$ concentrations tended to increase as $\mathrm{N}: \mathrm{K}$ ratios decreased. This suggests that the $\mathrm{N}: \mathrm{K}$ ratio may be more important than absolute amounts of $\mathrm{K}$ applied in determining foliar $\mathrm{K}$ concentrations.

Foliar Mg concentrations were also affected by fertilization treatment. The highest $\mathrm{Mg}$ concentrations were found in the smallest palms, suggesting a strong dilution effect (Tables 3 and 5). Nitrogen fertilization rate had a significant negative influence on foliar $\mathrm{Mg}$ concentrations, but did not significantly affect concentrations of N, P, or $\mathrm{K}$ (Table 6). On the other hand, $\mathrm{K}$ fertilization rate had a positive effect on foliar K concentrations, but negative effects on $\mathrm{Mg}$ concentrations. The negative effects of both $\mathrm{N}$ and $\mathrm{K}$ fertilization on $\mathrm{Mg}$ concentrations can be attributed to well-known dilution effects associated with $\mathrm{N}$ fertilization and $\mathrm{K}-\mathrm{Mg}$ antagonisms (Broschat et al., 2008; Jegananthan, 1990; Manciot et al., 1979; von Uexkull and Fairhurst, 1991).

In general, optimum growth and quality were obtained when using high rates of both $\mathrm{N}$ and $\mathrm{K}$ for areca and Mexican fan palms. The optimum application rates for $\mathrm{N}$ and $\mathrm{K}$ determined in this study (12.2 $\mathrm{g} \cdot \mathrm{m}^{-2}$ of $\mathrm{N}$ and $\mathrm{K}$ every 3 months) is about twice the rate recommended for palm maintenance in Florida landscapes, but equal to that suggested for field nursery production of palms where growth rate is also important (Broschat, 2014). Actual N and $\mathrm{K}$ application rates were found to be better predictors of palm quality than $\mathrm{N}: \mathrm{K}$ ratio at these high-fertilization rates typically used for field nursery production. However, at slightly lower application rates $\left(9.78 \mathrm{~g} \mathrm{~N} / \mathrm{m}^{2}\right)$, more typical of those used for landscape palm maintenance, palm quality of both species generally improved as the $\mathrm{N}: \mathrm{K}$ application ratio was decreased (Tables 1 and 3). Optimum treatments had $\mathrm{N}: \mathrm{K}$ ratios ranging between 0.5 and 1.0. This research supports current University of Florida recommendations for landscape palm fertilizers specify an $\mathrm{N}: \mathrm{K}$ ratio of 0.8 (Broschat, 2014).

This study was done on a calcareous sandy fill soil that is typical for landscapes in coastal cities in Florida and other southeastern states in the United States. Further research will be needed to determine optimum $\mathrm{N}$ and $\mathrm{K}$ fertilization rates for other soil types where palms are grown. This study was done using controlled-release sources for $\mathrm{N}$ and $\mathrm{K}$, and it is not known whether similar 
Table 6. Multiple regression equations for effects of $\mathrm{N}$ and $\mathrm{K}$ fertilization rates on nutrient composition of areca (Dypsis lutescens) $(n=74)$ and Mexican fan palms (Washingtonia robusta) $(n=76)$ growing in a calcareous sandy fill soil in Fort Lauderdale, FL.

\begin{tabular}{|c|c|c|c|c|c|c|c|c|}
\hline \multirow[b]{3}{*}{ Parameter } & \multicolumn{8}{|c|}{ Areca palm } \\
\hline & \multicolumn{2}{|c|}{$\mathrm{N}$} & \multicolumn{2}{|c|}{$\mathrm{P}$} & \multicolumn{2}{|c|}{$\mathrm{K}$} & \multicolumn{2}{|c|}{$\mathrm{Mg}$} \\
\hline & Coefficient & $P$ value & Coefficient & $P$ value & Coefficient & $P$ value & Coefficient & $P$ value \\
\hline $\mathrm{N}$ rate & -0.009 & 0.37 & -0.001 & 0.4 & -0.017 & 0.059 & -0.001 & 0.3 \\
\hline $\mathrm{K}$ rate & -0.011 & 0.13 & 0.003 & 0.005 & 0.03 & $<0.0001$ & 0.003 & 0.009 \\
\hline $\mathrm{N}$ rate $\times \mathrm{K}$ rate & 0.001 & 0.11 & -0.0002 & 0.1 & -0.0003 & 0.65 & -0.00004 & 0.72 \\
\hline Intercept & 1.307 & $<0.0001$ & 0.1 & $<0.0001$ & 0.537 & $<0.0001$ & 0.15 & $<0.0001$ \\
\hline \multirow[t]{3}{*}{ Model } & \multicolumn{2}{|c|}{$R^{2}=0.04, P=\mathrm{NS}$} & \multicolumn{2}{|c|}{$R^{2}=0.18, P=0.003$} & \multicolumn{2}{|c|}{$R^{2}=0.42, P<0.0001$} & \multicolumn{2}{|c|}{$R^{2}=0.20, P=0.002$} \\
\hline & \multicolumn{8}{|c|}{ Mexican fan palm } \\
\hline & \multicolumn{2}{|c|}{$\mathrm{N}$} & \multicolumn{2}{|c|}{$\mathrm{P}$} & \multicolumn{2}{|c|}{$\mathrm{P}$} & \multicolumn{2}{|c|}{$\mathrm{Mg}$} \\
\hline Parameter & Coefficient & $P$ value & Coefficient & $P$ value & Coefficient & $P$ value & Coefficient & $P$ value \\
\hline$\overline{\mathrm{N} \text { rate }}$ & 0.015 & 0.25 & 0.002 & 0.21 & 0.009 & 0.57 & -0.006 & $<0.0001$ \\
\hline $\mathrm{K}$ rate & -0.002 & 0.82 & 0.002 & 0.06 & 0.058 & $<0.0001$ & -0.004 & 0.0001 \\
\hline $\mathrm{N}$ rate $\times \mathrm{K}$ rate & -0.0008 & 0.47 & -0.0003 & 0.023 & -0.003 & 0.025 & 0.0004 & $<0.0001$ \\
\hline Intercept & 1.641 & $<0.0001$ & 0.198 & $<0.0001$ & 0.956 & $<0.0001$ & 0.186 & $<0.0001$ \\
\hline Model & \multicolumn{2}{|c|}{$R^{2}=0.03, P=\mathrm{NS}$} & \multicolumn{2}{|c|}{$R^{2}=0.08, P=\mathrm{NS}$} & \multicolumn{2}{|c|}{$R^{2}=0.37, P<0.0001$} & \multicolumn{2}{|c|}{$R^{2}=0.30, P<0.0001$} \\
\hline
\end{tabular}

NS $=$ nonsignificant

results would have been obtained using more water-soluble sources.

\section{Literature Cited}

Broschat, T.K. 1979. Principal component analysis in horticultural research. HortScience 14:114-117.

Broschat, T.K. 1990. Potassium deficiency of palms in south Florida. Principes 34:151-155.

Broschat, T.K. 2009. Palm nutrition and fertilization. HortTechnology 19:690-694.

Broschat, T.K. 2014. Fertilization of fieldgrown and landscape palms in Florida. Univ. Fla. Environ. Hort. Publ. ENH-1009. 15 Sept. 2015. <http://edis.ifas.ufl.edu/ep261>.

Broschat, T.K. 2015. Fertilization of landscape palms to reduce nitrogen and phosphorus impacts on the environment. HortScience 50:469-473.
Broschat, T.K. and M.L. Elliott. 2009. Effects of fertilization and microbial inoculants at transplanting on the growth of Mexican fan palm and queen palm. HortTechnology 19:324-330.

Broschat, T.K., D.R. Sandrock, M.L. Elliott, and E.F. Gilman. 2008. Effects of fertilizer type on quality and nutrient content of established landscape plants in Florida. HortTechnology 18:278-285.

Hach, C.C., B.K. Bowden, A.B. Koplove, and S.V. Brayton. 1987. More powerful peroxide Kjeldahl digestion method. J. Offic. Anal. Chem. 70:783-787.

Jegananthan, M. 1990. Studies on potassiummagnesium interaction in coconut (Cocos nucifera), p. 611-617. In: M.L. Van Beusichem (ed.). Plant nutrition-Physiology and applications. Kluwer Academic Publ., Alphen aan den Rijn, the Netherlands.
Manciot, R., M. Ollagnier, and R. Ochs. 1979. Mineral nutrition and fertilization of the coconut around the world. II. Study of the different elements. Oleagineux 34:563-580.

Mills, H.A. and J.B. Jones, Jr. 1996. Plant analysis handbook II. MicroMacro Publ., Athens, GA.

Ogden, R.F., F.A. Pokorny, H.A. Mills, and M.G. Dunavent. 1987. Elemental status of pine barkbased potting media. Hortic. Rev. 9:103-131.

Poole, R.T. and C.A. Conover. 1985. Nitrogen, phosphorus, and potassium fertilization of Brassaia actinophylla, Calathea makoyana, and Chrysalidocarpus lutescens. J. Environ. Hort. 3:1-3.

Tisdale, S.L. and W.L. Nelson. 1975. Soil fertility and fertilizers. Macmillan, New York, NY.

von Uexkull, H.R. and T.H. Fairhurst. 1991. Fertilizing for high yield and quality in the oil palm. Intl. Potash Inst., Berne, Switzerland. 